الاتحاد الاوروبي والمشكله القبرصيه: إثنكاليه العلافه الاوروبيه القبرصيه

وليا محمود احمد*

مقدمها

لا تزأل مساله ضم هبرص بشطرها الجنوبي إلى الاتحاد الاوروبي في ايار عام

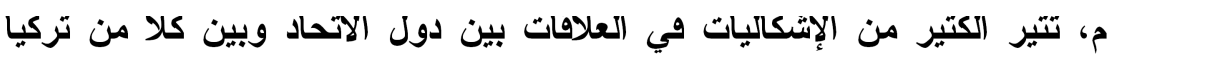
و اليونان. ويرى بعض الابلوماسيين الاوروبيين، إن الاتحاد الاوروبي ربما و اخطا حينما واثق على هبول عضويه وبرص، بينما الجزيرة لازالت مقسمه. بينما يرى محللون سياسيون اوروبيونه إن الاتحاد الاوروبي لم يجاتب الصواب ي سياسته تجاه وبرصه

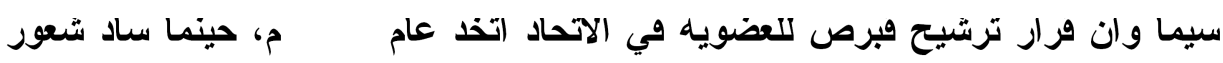
في اوروبا انداك مفاده ان تركيا تعمل على عروله التوصل إلى تسويه مقبوله بشنان الجزيرة القبرصيه المقسمه مند العام I9V مه اتر الغزو العسكري التركي لشمال الجزيرة.

$$
\text { الموفع الجغراهي والجدور التاريخية لجزيرة صبرص }
$$

تقع جزيرة هبرص في اوصى شرق البحر المتوسط، وهي تالت كبريات الجزر في هاا البحر جنبا إلى جنب مع جزيرتي صقليه وسردينيا، تبعد عن الساحل السوري حوالي

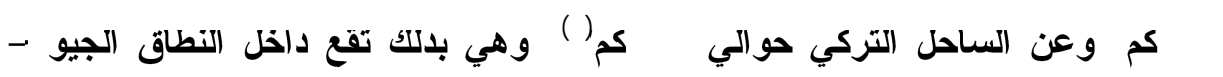
إستر اتيجي لكل من سوريا ولبنان وإسرائيل، وهدا جعلها ساحه خلقيه للصر اع

* مدرس مساعد، فس الارسات السياسيه والاستر اتيجيه، مركز الاراسات الاوليميه، جامعه الموصل، 
العربي - الإسرائيلي، حيث شهرت الجزيرة مواجهات داميه بين الفلسطينين والاسر ائيايين (r).

تعود جدور النزاع في جزيرة وبرص إلى عام IOVI م حينما غزا العتمانيون

الجزبرةالماهوله انداك باليوناتيينه وبدات هجرة تركيه من الآناضول إلى الجزيرة، وشتل هؤلاء الاتراك المهاجرون مع القبارصه الدين اعتنقوا الإسلام، اصل القبارصه الاتراك

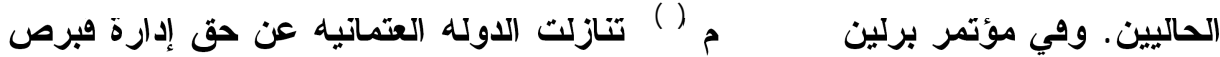
لبريطانيا، وفي كانون الاول ع|91 م ضمت بريطانيا الجزيرة إلى ممتكاتها، اتر إعلان الدوله العتمانيه الحرب على الحلفاء بعد اندلاع الحرب العالميه الاودىعام عا9 ا مه وفي تموز 19 م م اعتروت تركيا بضم وبرص إلى بريطانيا، وفي اب •197 م تم إعلان استقلال وبرص كجمهوريه واصبح رئيس الاساصفه مكاريوس التالت (أفبرصي يوناني)، رئيسا للجمهوريه، في حين اصبح هاضل كجوك((وبرصي تركي) نائبا للرئيس، وضمنت كلا من بريطانيا وتركيا واليونان هلا الاستقلال ووحدة الدوله الجديدة (ع). لم يكن هلا الاستقلال كاملا بقعل التلخل الخارجي في شؤون الجزيرة مسن جهــهـ ومن جهه اخرى، حمل الاستور القبرصي في داخله بدور المشاكل والازمات، مما جع لل الجزيرة حديته الاستقلال عرضه للمشاكل، وبالفعل وقل توالت عليها الازمات في الاعــوام

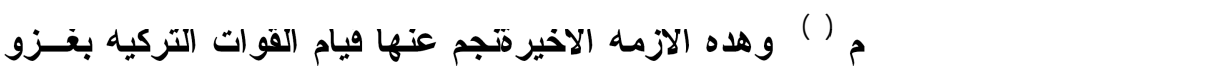
الجزء الثمالي للجزيرة، الامر الدي ادى إلى تقسيم الجزيرة إلى فسمين (لفبرصي تركي، ووبرصي يوناني)، ومند دلك الحين والمشكله القبرصيه لها حضور شبه دائم في المحاهل الاوليه، وتجدد دلك الحضور إبان انضمام الثطر الجنوبي مــن الجزيــرة إلــى الاتحسـاد الاوروبي عام ع.-م م ، مما اتار تعقيدات وي داخل الاتحاد جاء فسم منها مسن روضـ القبارصه الاتراك لفكرة ان يتولى القبارصه اليوناتيين لوحدهم تمتيل جمهوريه فبرص في

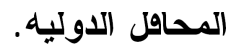


اوروبا والعلافه مع فبرص

بعد انضمام الجزء الجنوبي من جزيرة وبرص إلى الاتحاد الاوروبي في

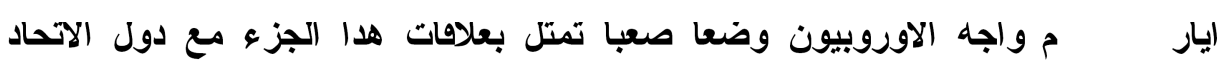
الاوروبي من جههه والوضع القاتوني غير المحسوم بالنسبه للجزء الشمالي من الجزيرة، من جهه تانيه. هدا الوضع الصعب الدي واجهه الاوروبيون ناجم ايضا عن ماهيه العلاقه التي تربط الجزء الشمالي من الجزيرة مع تركيا، التي بلورها لا تعترف بسلطه الحكومه القبرصيه وي الجزء الجنوبي من الجزيرة، لكنها بنقس الوهت تعترف بسلطه الحكومه القبرصيه التركيه في شمال الجزيرة، في حين لا تعترف حكومه الجزء الجنوبي بسلطه الحكم في الجزء الشمالي (7). ها الامر انعكس برمته على مستقبل العلافه بين تركيا والاتحاد الاوروبي من ناحية، وعلى مستقبل العلاهه بين اليونان الداعمه لسلطه الحكومه القبرصيه في الجزء الجنوبي من الجزيرة، وبين الاتحاد الاوروبي عندما يتعلق الامر بمساله انضمام تركيا إلى الاتحاد من ناحيه تانيهه إد انه بامكان الدوله العضو في الاتحاد استخدام (القيتو) ضد التد ترشيح اي دوله لنيل العضويه في الاتحاد وهلا ما تخشاه تركيا من احتمال استخدام اليونان او هبرص الجنوبيه لهـا الحق، الامر الدي اتار مخاوف الولايات المتحدة الامريكيه على مستقبل حليقتها تركيا، وجعل الامريكان ياخلون في هله المسالهه إد ترغب واشنطن في ان تنضم تركيا إلى الاتحاد الاوروبي، في حين رد ممتلو الاتحاد بالقوله ان محادتات

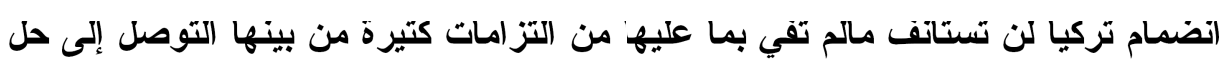
للمشكله القبرصيه بما ينهي احتلال الجيش التركي للجزء الثمالي من الجزيرة (v). تشكل المشكله القبرصيه نقطه (خلاف نادرة) بين الولايات المتحدة وبريطانيا، وهده الاخيرة مع إضفاء الطابع اليوناني على الجزيرة، بينما تؤيد الولايات المتحدة تركيا في موفها تجاه الجزيرة، لان الامريكان ينظرون الي المشكله نظرة عسكريه بحته لدلك تبنت 
و اشنطن الحل الكونقدرالي الاي يضمن للقبارصه الاتراك كامل حقوههم، ويضمن وجود حاميه عسكريه تركيه وي الجزيرة لقطع الطريق على التقلفل الروسي في الجزيرة، الدي يراهن على الرابطه الطائفيه ميع القبارصه اليونانيين الارتدوكس ي حين يؤيد الاتحاد الاوروبي حلا ودراليا محدودا وي الجزيرة ())، من خلال تطبيق استراتيجيه خاصه بها تتضمن مساعدة وبرص على زيادة كفاعة إدارتها ومحاكمها العامة، وبالتالي يمكنها المشاركه وي برامج المجتمع الاوروبي وان تصبح مرتبطه بعمل مؤسساته الخاصه. ولم يغقل الاتحاد فضيه الجاليه القبرصيه التركيه من خلال تحقيق مبدا النفع العام لكل الجماعات الاتنيه والعرويه المكونه لمجتمع الجزيرة، والمساهمه في تحقيق السلم

و المصالحة، وان يكون هناك تمتيل للقبارصه الاتراك وي الووهود الرسميه القبرصيه(9).

خطه الامم المتحدة لحل مشكله الجزيرة ضضت خطه الامم المتحدة التي اقترحها الامين العام السابق للامم المتحدة كوفي عنان في تثرين التاني r. -.r، بإقامه دوله كونفلراليه على غرار النمودج السويسري بمجلس رئاسي يضم ممتلي الجاليتين القبرصيتين ويتناوب اعضاؤه على الرئاسه وتعيد الخطه رسم المناطق الخاصه للجاليتين، وهو ما تتحفظ عليه تركيا، حيث ستتقلص مساحه القبارصه الاتراك إلى Aه\% من مساحه الجزيرة بدلا من Aس\% تخضع لسيطرته حاليا، كما تضع الخطه جدولا زمنيا وعدديا لإعادة توطين عدد من القبارصه اليونانيين نزحوا من مناطق سيطرة القبارصه الاتر الك (- (). الهم ملامح مشروع الامين العام السابق الدي عرضه على زعيمي القبارصه الاتر الك واليوناتيين كان عنواته ل(سس لحل شامل للمشكله القبرصيه)، تمل بالاثي: اولا: شكل الدوله: اهترح المشروع ان يكون اسم الدوله "(الجمهوريه القبرصيه المتحدة)،

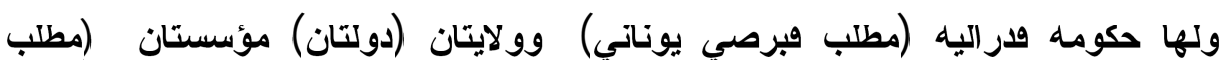
هبرصي تركي). ثلنيا: السيادة: اوضح المشروع ان العلافه بين الحكومه القدراليه والولايات المؤسسه سيكون مماتلا للوضع في سويسرا، وان الجمهوريه القبرصيه المتحدة كيان سياسي دو 
سيادة واحدة وبنقس الووت تمارس الولايات المؤسسه كل السلطات غير الموكله للحكومه القدر اليه. ثالثا: الرئاسه: طرح المشروع إنشاء مجلس رئاسي يتكون من (7) اعضاءه اتتين من القبارصه الاتراك والاربعه الاخرين من القبارصه اليونانيينه ويكون منصب الرئيس بالتتاوب بين الاعضاء الستهه على ان يتم اتخاد القرارات في المجلس الرئاسي بالإجماع هلر الامكانه او بالاعلبيه البسيطه، على ان تضم تلك الاغلبيه على الاول عضوا هبرصيا

$$
\text { تركيا وعضوا وبرصيا يوناتيا. }
$$

رابعا: السلطه التشريعيه، (البرلمان القدرالي): افترح المشروع ان يكون البرلمان تتائيا

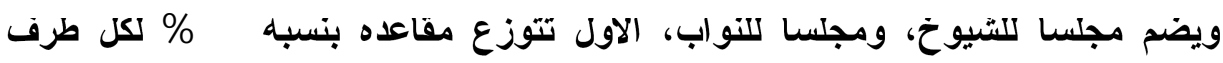

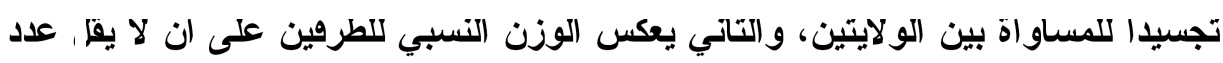
المقاعد المخصصه للولايه الواحدة عن \%0\% من عدد المقاعد الكليه. خلمسا: توزيع الاختصاصات: بين المشروع الاختصاصات الخاصه بالحكومه القدراليها. واللازمه لكي تتحرك فبرص وتتكلم بصوت واحد في الاتحاد الاوروبي، ولكي تحمي وحتها وحدودها ومواردها وتر اتها، كما تعرض المشروع الي الهيه وجود محكمه عليا

$$
\text { للاوله (11) }
$$

هام الامين العام مشروعه هدا إلى زعماء القبارصه الاتراك والقبارصه اليونانيين في ادار ع - .'r م وطلب منهما عرضه على الاستفتاء وي نيسان من نفس العامه ورض زعيم القبارصه الاترالك دلك وانسحب على إتره من المقاوضاته الامر الدي اكد المخاوف التركيه(حكومه اردوغان)من الاتتخابات العامه البرلمانيه التي اجريت في فبرص التركيه

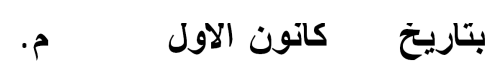

إن علم تحقيق نتائج حاسمة من جاتب القوى التي تعول عليها الحكومه التركيه لاجل إعادة توحيد الجزيرة، بددت الاحلام التركيه في تحقيق نتيجه ايجابيه في مفاوضات 
توحيا الجزيرة، وقوى المعارضه الحزبيه القبرصيه التركيه، ضد الزعامه التقليديه (رؤوف دنكتاش) المدعوم من الجيش التركي، لم تتجح وي الحصول على ورصه الجلوس على مائدة المفاوضات والتوفيع على خطه توحيد الجزيرة بما يتو اقق مع الرؤى اليونانيها. إد ماز الحزب الجمهوري المعارض أيسار الوسط) بزعامه محمد علي طلعت إلرئيس الحالي لقبرص التركيه)، بالمرتبه الاولى، لكن عدم وززه بالاغلبيه اللازمه لتثكيل حكومه بمقرده اتار خيبه امل وي تركيا ودوعها إلى العودة مجددا إلى دعم دنكتاشه بعد ان كاتت تميل لخيار إبعاده عن الفعل السياسيه ومطالبتها بتثكيل حكومه وطنيه وبرصيه تركيه تضم كل الاحزاب السياسيه البرلمانيه. لقد انعكس المازق السياسي للمعارضه القبرصيه التركيه المدعومه من الحكومه التركيه الحاليها اتعكس سلبا على مفاوضات توحيد الجزيرة بانسحاب دنكتاش من تلك المفاوضات(Ir)، مما دوع الامم المتحدة إلى إجراء استقتاء مباشر على خطه الامين العام في شطري الجزيرة في ع نيسان ع - ."امه هبيل انضمام الثطر الجنوبي إلى الاتحاد الاوروبي، الدي كان مقررا في الاول من ايار ع - ."امه على امل تحقيق اختراق في القضيه باستتمار جدول اعمال الاتحاد الاوروبيه و التي لا تتعلق بقضيه انضمام الجمهوريه القبرصيه للاتحاد وقطه بل وانضمام تركيا ايضاه غير إن النتائج السلبيه التي جاءت من الشطر اليوناني افشلت عمليه الاستفتاءه وضد كتب الامين العام للامم المتحدة حينها، شهادته في تقريره عن اعمال المنظمه فائلا: "يؤسفني ان اهيا بانه ورغم الممارسات المكتقه لمساعي الحميدة، هان مشكله قبرص ماز الت بغير حل. لقد تبددت رصه وريدة للتوصل إلى تسويه تفتح المجال لتوحيا الجزيرة وبل انضمامها إلى الاتحاد الاوروبي. وإدا كان من شان اي تسويه ان تتم هبل انضمام وبرص اليوناتيه للاتحاد، صاتها ينبفي ان تتيح لقبرص المعاد توحيدها سبيل الانضمام إلى الآحاد الاوروبي، كما إنتي لا اتصور ان تمه صائدة ترجى من خلال اتخاد مبادرات جديدة، الا إدا دلل الطرهان على التزامها بتسويه النزاع الدي طال امدة"(r). 


$$
\text { إثكاليه العلاقات الاوروبيه - القبرصيه }
$$

لقد ترك انضمام فبرص اليونانيه إلى الاتحاد الاوروبي اترا سلبيا عل مسقل العلافه بين الاتحاد وفبرص من ناحيه وبين الاتحاد وتركيا من ناحيه تاتيه. وعلى مستوى الاتيل العلاهه بين الاتحاد وفبرص وقد تعقدت الامور داخل الاتحاده واشر رض القبارصه اليوناتيين لخطه الامم المتحدة المشار إليها، نقطه سلبيه وي سجل فبرص اليونانيه، بينما عسل فبول القبارصه الاتراك للخطه اعلاه نقطه ايجابيه لصالحهم لم يتمكن الاتحاد من تجاهلها مما اوفعه وي حرج عندما ضم وبرص إلى عضويته، وليس من وبيل المبالغه القوله بان الاتحاد الاوروبي يعد واحدا من المسؤولين عن استمرار العزله الاقتصاديه للقبارصه الاتراكه وربما كأن من الاوضل الاتتظار حتى تتم تسويه المشكله القبرصيه وضم الجزيرة كامسه إلى عضويته (ع) . ورغم ان اعضاء الاتحاد تعهدوا بتقليم المساعدات الاتئ الماليه إلى القبارصه الاتراكه إلا انه ليس واضحا فيما إدا كان دالكك سيكون كاصيا لإرضائهمه حيث يريد القبارصه الاتراك استئناف رحلات الطيران بثكل ووري إلى جنوب هبرص وتصدير منتجاتهم بشكل مباثر إلى اوروبا، لكن هله الرغبه القبرصيه التركيه ستواجها عقبه هاتونيه لان الاوروبيون حتى وان رغبوا وي إنهاء عزله الشمال القبرصي، إلا انه من الصعب روع الحظر التجاري بشكل كامل عن شمال الجزيرة المفسمة لان دلك يتطلب فرارا من محكمه العدل الاوروبيه(10). بالقلال، يبدو الجنوب القبرصي اليوناني المزدهر افتصاديا، غير عابئ للاتتقادات، وغير عابئ بالتحديرات من انه سيقابل بجفاء داخل الاتحاد اتر روضه لخطه الامم المتحدة المشار إليهاه وينظر القبارصه اليونانيون إلى القلق الدولي على صبرصه (على اته ضغط دولي غير مقبول) (17)، غير ان الرئيس القبرصي الحالي (تاسوس بابا دوبولص)، اعلن بان الحكومه القبرصيه في الجنوب ستضمن استفادة القبارصه الاتراك 
من مزايا الاتحاده ووعد بان القبارصه اليونانيين لن يتخلوا عن القبارصه الاتراك، لكنه بالمقابل لن يقبل ابلا الاعتراف بلوله الشمال، وهو الموفف الاي يشاطره الراي ويه مسؤولون في الاتحاد الاوروبي، الدين رددوا باتهم سيارسون كل شئ عدا الاعتراف الرسمي بجمهوريه وبرص الشماليه (IV) اما على مستوى العلاوه بين الاتحاد الاوروبي وتركيا بشان المشكله القبرصياه وقد ازدادت تعقيداء وبعد انضمام الجزء الجنوبي القبرصي اليوناني للاتحاده اصبح واضحا ان القوات التركيه في شمال وبرص، هي ووات اجنبيه تحتل جزء من اراضي احدى دول الاتحاد، هلده الإنتكاليه حاولت تركيا تفاديها حتى لا يضطر الاتحاد إلى الطلب من تركيا الآسحاب عسكريا من شمال الجزيرة وهلا مالا ترغب تركيا بتقيده علىالاول وبل اتضمامها للاتحاد، ويعتقد الاتراك باته لن يكون هناك تحرك اوروبي ايجابي من الاتحاد نحو انقرة مالم يحصل انفراج وي المشكله القبرصيهه ويرى بعض المسؤولين الاتراك إن الاتحاد الاوروبي ود اصبح مقيد اليدين تجاه حل المشكله، لأنه اضحى طرها وي النزاع على الجزيرة بعد ضمه للشطر الجنوبي من وبرصه وان المشكله القبرصيه هي بالنسبه للكتيرين من مسؤولي الاتحاد دريعه يعللون بها موفهم المناوئ

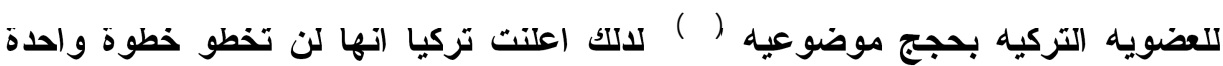
باتجاه (التكامل السياسي) مع القبارصه الاتراكه إلا بعد ان يضم الاتحاد الاوروبي، القبارصه اليوناتيين إلى عضويته. بالمقابل هادت اليونان باستخدام حق النقض لوهف مشروع توسيع الاتحاد|(الدي تستقيد منه تركيا) في حاله عدم التوصل إلى تسويه تعترف بحقوق القبارصه اليونانيين. وعلى الرغم من كترة المحادتات بين الطرهين المتنازعينه الاانهما مازالا على عنادها ، ويما حدر مسؤولون في الآحاد، تركيا من تجاهل حقوق القبارصه اليوناتيين، او ضمها لشمالي وبرص (19). هده التحديرات ساعدت على زيادة تعقيدات الوضع في الجزيرة المقسمه، واتحى الرئيس القبرصي التركي باللانمه على الاتحاد الاوروبي وحمله مسؤوليه تفام الوضع الحالي في الجزيرة بسبب اتحيازه للقبارصه اليونانيينه ورغم الاتفاق الدي تعهل بموجبه 
زعماء القبارصه الاتراك واليوناتيينه بالاستمرار على إجراء اللقاءات والمشاورات، لكن الجانب القبرصي اليوناني يتهرب من تعهذاته ووعوده للمنظمه الدوليه، وهو يارك ان الاتحاد الاوروبي سيدعمه في جميع الحالات (-r)، وهدا واضح لان القبارصه اليونانيين ليس لايهم ما يخسرونه وهم يعتقدون إتهم يملكون كل شئه وهوق دلك وقل اصبحت عضويه وبرص اليوناتيه في الاتحاد اداة لتعطيل المبادرات الاوروبيه المتعلقه بالمشكله القبرصيه، وحكومه الثطر الجنوبي اصبح في مقدورها استخدام حق النقض ضد اي تحرك من اوروبا لا ينسجم ومصاحها وحساباتها، والاهم من دلك ودرتها على عرهله مسار تركيا الاوروبي (II). إن المشكله القبرصيه وي العلاهات بين الاتحاد الاوروبي وتركيا هي وليدة عقود طويله، والإطراف التلاته لازالت بعيدة عن اي تسويه، وستبقى مشكله اللاجئين، و المثاركه وي السلطه حجر عترة وي العلاهات الاوروبيه - التركيه (Tr).

$$
\text { مستقبل التسويه في فبرص }
$$

يرى هسم من المسؤولين القبارصه اليوناتيين إن إنشاليه النزاع هي فبرص لابد ان تعالج سياسيا وبمساعدة الامم المتحدة، هالموف على الارض يتجاوز الموافف السياسيه المتشنجه التي يطلقها الساسه، وعلى سبيل المتال لم تتمكن حكومه القبارصه اليونانيين من وفف عمليات التبادل التجاري ميع سكان الجزء الثمالي من الجزيرة، مما شجع وزراء خارجيه دول الاتحاد الاوروبي على الحوار بخصوص إقامه تبادل تجاري مباتُر مع الشطر الشمالي، ويامل الدبلوماسيون الاوروبيون، ان يساعد هدا التحرك،

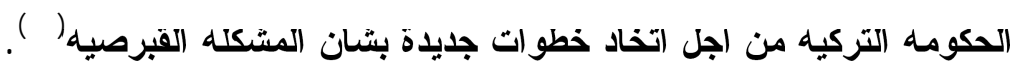
اما على الجاتب الاخر، هلا يزال الزعيم القبرصي التركي محمد علي طلعث يرى ان الحل لن يكون إلا من خلال دوله واراليه تضم الكياتين القبرصيينه ويكون التمتيل السياسي 
القبرصي التركي مشابه للتمتيل السياسي القبرصي اليوناني، لان القبارصه الاتراك ليسوا بأفلية، وروض الربط بين وبرصه والعلاهه بين تركيا والاتحاد الاوروبي (عَ). إدن، توجد ظروف معقدة ومتشابكه تحيط بالقضيه، وهناك من يرى بان الحلول

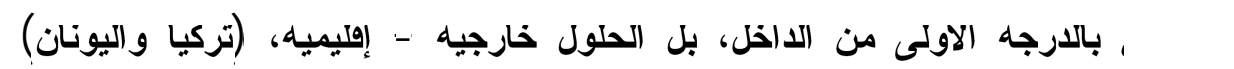
ودوليه (الولايات المتحدة). ورغم اتفاق تركيا واليونان على تخفيف التوتر بينهاه إلا ان لهن عدم الاستقرار الإقليميه والاستقطاب الإسلامي والعلماني في تركيا، وضعف البنيه السياسيه وي اليونان، وضلا عن اهتمام الحكومتين التركيه واليونانيه بتدعيم مركزهما الداخليه كلها امور لم تؤدي إلى التوصل لحل وسط يرضي الاطر اف المتنازعله والارجح

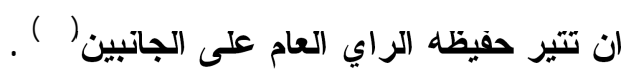
غير ان هناك رايا اخر يقول بان الحلول تاتي من الاخل بالدرجه الاساسه حيث يعتقا.

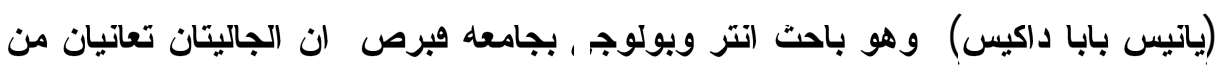
حاله اسمها (التوحد العروي)، وان المصابين بها الحاله هم من الاين ينقصلون عن و اقعهم ولا يمكنهم الإصغاء او التخاطب مع الدين من حولهه، ويشرح المقارنه فائلا، بانه يعتقل ان الوضع في وبرص ينطوي على انغلاق كامل من كل جانب على مشاعره ويشعر بانه ضحيه للاخر، وبالتالي لا يستطيع ان يخرج خارج دائرة نقسه ليستمع إلى الام ومعاناة الاخر. وإدا كان الامر كللك فان وص المصالحه ولعادة توحيد الجزيرة في المستقبل المنظور تبدو بعيدة المناله حتى وان وبلت الجاليتين في نهايه الامر بخطه ما لإعادة توحيد الجزيرة(r7). خاتمهل و استتتاجات

يبدو من الواضح ان الآحاد الاوروبي ربما يكون ف اخطا عندما اعتبر ان الاستفتاء النعبي -على خطه إعادة توحيد الجزيرة شطري فبرصه والمقده من امين عام الامم المتحدة السابق كوفي عنان - هو النتيجه التي يمكن الركون لها عند البحث عن تسويات او حلول للمشكله القبرصيه، وقل جائت النتائج تعكس مستوى تفكير الجاتب القبرصي، الدي رض خطه إعادة الجزيرة، وعلى الرغم من ان الاتحاد عبر عن رغبته 
في ضم الجزيرة كامله مراهنا على الاستقتاءه إلا ان هله الرغبه لم تلق بالا لاى القبارصة اليونانيين، وبلاو وكاتهم متاترين بالرابطه الارتدوكسيه التي تغليها الكنيسه القبرصيه. اكتر من تاترهم بالرابطه الوطنيه ونظروا إلى القبارصه الاتراك على اتهم اهليه وليسوا بمكون تان لسكان الجزيرة. هله الإشكاليه فشل الاتحاد الاوروبي في التعامل معها فما الدي حصل؟ الحقيقه ان الدي حصل وعلا، هو ان الاتحاد الاوروبي اورث نفسه وضيه استعصت على ولى الحل طيله اربعه ورون، وجعل من مشكله وبرص عبئا على الدول الاعضاء. ان حل

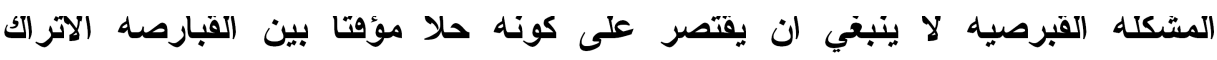
والقبارصه اليوناتيينه بل ينبفي ان يتسع ها الحل ليشمل تمكين الجزيرة من ان تصبح طرها فاعلا إلفيميا ودوليا، ويما يقضي على ايه تداعيات داخليه في الجزيرة ف تؤتر على

الاتحاد الاوروبي برمتهه هما هو الدور الدي تلعبه دوله مجزاة وعاصمتها مجزاة ايضا؟ لاثشك ان عضويه هبرص اليونانيه في الاتحاد لها تاتير سلبي على مسيرة تركيا الاوروبيهـ. إد بمقدرها الاعتراض على عضويه تركيا، هلا فضلا عن إحراجها، وعمليه ضم وبرص للاتحاد ود تترتب عليها مواجهه مع تركيا، وهـه الاخيرة لايها ووات عسكريه في شمال الجزيرة، وكيف سيتصرف الاتحاد مع دوله من غير اعضائه تحتل جزء من اراضي دوله هي عضو فيه؟ لقد حاولت تركيا تجنب هده الحاله عندما حاولت الضغط على هادة القبارصه الاتراك لإعادة توحيا الجزيرة لتوير ورصه لخروج القوات التركيه، حتى لا يشكل دلك دريعه للاتحادير ان نتائج الاستقتاء سجلت موها ايجابيا يحسب لصالح الاتراك (ضنمنيا)، مما عزز من رصيد تركيا التقاوضي ميع الاتحاد. ول يكون هناك امر اخر وراء ضم هبرص للعضويه في الاتحاد، وهو الرغبه الاوروبيه في دوع النقود الامريكي والروسي عن الجزيرة، وفضل الاوروبيون احتواء 
الجزيرة من خلال المظله الاوروبيهه بلالا من ان يتم احتو ائها تركيا (الولايات المتحدة)، او

يوناتيا أروسيا).

تمه امر يمكن استتتاجها وهو ان البناء الداخلي لاي مجتمع هو الحصانه الاكيدة

بوجه اي تلخل إقليمي او دولي، وحقيقه يجب ان تقال بها الثشانه ان الحلول الممكنه للوضع في هبرص يجب ان تتبع من الداخل القبرصيه هعلى القبارصه اليوناتيين ان لا يتجاهلوا حقوق القبارصه الاتراك، ان لا يتعاملوا معهم على اساس انهم (فليه وبالتالي يعطون لاتفسه وقط حق إدارة البلاد، وبالمقابل، على القبارصه الآر الك ان يتفهوا حقيقه ان الجزيرة ليست ملكا لهم وقطوان العتمانيين عندما غزو الجزيرة عام LVI Iان غالبيه سكانها من اليونانيين وان على الطائقتين ان تتخلصا من مرض اسمه (الوطن الام)، وان عليهم ان ينظروا لاتفسهم على انهم وبارصه اولا واخيراه هده الامور كان ينبغي على الاتحاد الاوروبي التركيز عليها عندما فرر ضم هبرص إلى عضويتها وان الاستمرار في تجاهل هاه الامور يضع العراهيل في العلاهات التي تحكم الاتحاد مي فبرص من ناحيها والعلاهات مع تركيا من ناحيه تاتيه. كما يمكن استتتاج امر اخر مما تقلمه وهو انه ينبفي ان تؤخد معايير مدى تقدم الثعوب وكريا وحضاريا بما يمكنها من التواصل الحضاري والفكري مع بقيه شعوب وامم العالمه عند تعلق الامر بمساله ضم القبارصه اليوناتيين للعضويه الاوروبيهه إد اتبت القبارصه اليوناتيون انهم ليسوا على درجه كايها من الوعي تمكنهم من ان يلعبوا دورا فاعلا وي عضويه الاتحاد الاوروبي، فليست العبرة في ضم الدوله او الكيان بل العبرة من ملى فاعليه هدا العضو او دالك في تجمع يعد الاهم في العالم تملنا وتطورا وهو الاتحاد الاوروبي، والحقيقه ان دلك يشكل نقطه في سجل الاتحاد وسينعكس سلبا على علاهه الاتحاد الاوروبي بقبرص بكلا شطريها. تمه استتناج اخير بها الثنان مفاده'، ان القبارصه فشلوا في فهم انتمائهم لجزيرتهم وكان على الاتحاد الاوروبي ان يتتبه لها الامر ويتانى في مساكه ضم الجزيرة إلى عضويته لكنه اخطا وي حساباته ايا كاتت وباتتالي ورث عبئا على دبلوماسيته ولامدا طويل. 
[rIr] [

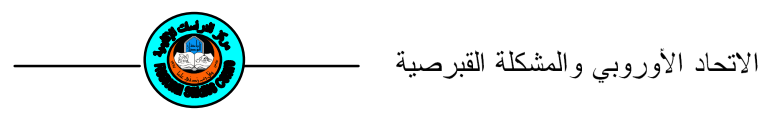

\title{
European Union And the Cyprus Question: The problem of Europe syprus Relations
}

\begin{abstract}
Waleed Mahmood Ahmed*
Abstract

The Europeans had tried to get use of United Nations plan to solve the Cyprus question in away to prepare the unification of the island since 1974 But the Greek Cypriots lost that chance. While the E.U. obligated itself with the promise given to unify the island by the date May 2004. The E.U. did not obligate it self to find out any solution for the issue.

The E.U. made Turkey responsible for any obstacle facing the issue. Therefore, Europeans were facing a reality representing a divide state in their union. Will the Europeans pressure succeed upon Turkey in unifying the last divided capitals inside Europe?
\end{abstract}

*Assist. Lecturer, Regional studies center. 
( ) هاشم خضير الجنابي: جغرافية اوراسيا ـ دراسة في الجغرافيا العامة والإقليمية،

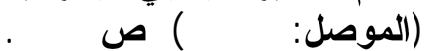

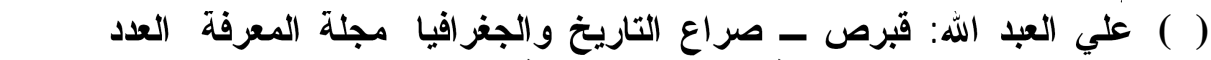
ع - -.r، منشور على شبكة المعلومات الاولية، على الموقع الاكتروني:

http://www.almarefah.com.article.php.id=

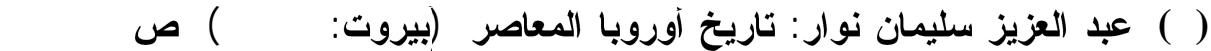

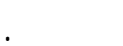

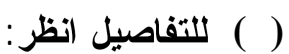

Question of Cyprus - Documentary Story 1960 - 1988 ,

(Cyprus:1988), p3

( ) للتفاصيل عن الأزمات القبرصية الثلاث انظر: وليا محمود أحمد: المشكلة القبرصية

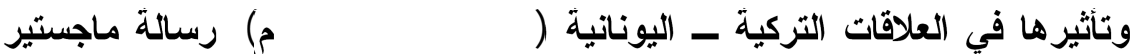

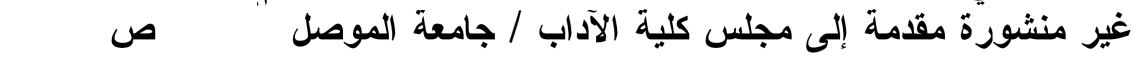

( ) للتفاصيل انظر الموقع الاكتروني الآتي على شبكة المعلومات الاولية:

www.Islamonline,net/arabic/news/2006-6/20/04/htm

( ) تابيثا مورغان:الحلم المؤجل، قبرص عشية الاستفتاء، بي بيسمي نيقوسيا، منشور على شبكة المعلومات الاولية، على المولى الموقع الاكتروني:

www.B.B.C.Arabic.com.22,April,2004.22:11GMT ( ) ) ملي العبد اللها المصدر السابق.

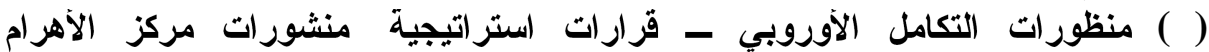

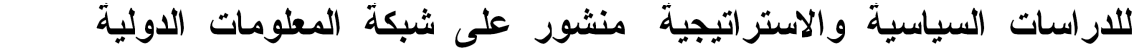
الموقع الاكتروني:

http://www.ahram.org.eg/.

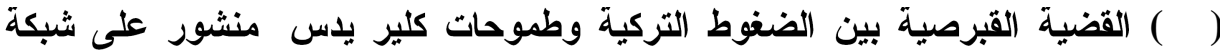

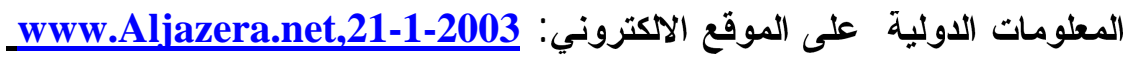

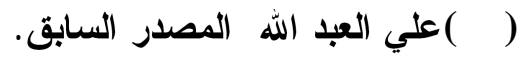

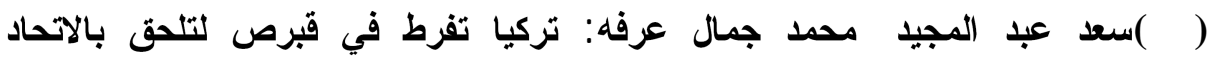

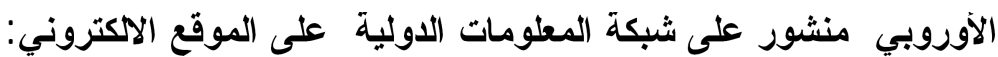

www./Slamonlin.net./Arabic/shariha/corner/index/shtm

(13) United Nations Docunment:No.A/58/1.

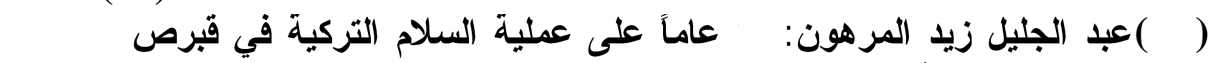

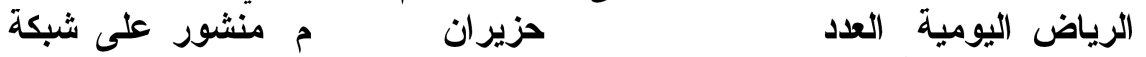
المعلومات الاولية ، على الموقع الإكتروني: http://www.alriyad.com/2007-6-29,19:55GMT 
( ) الاتحاد الآوروبي يقرر مساعدة القبارصة الآثرالك، منشور على شبكة المعلومات الاولية، على الموقع الأكتروني:

www.B.B.Arabic.Com.26April-2004,19:55.GMT

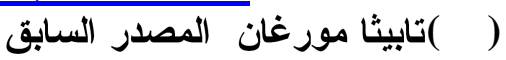
( ) (الاتحاد الأوروبي يقرر مساعدة القبارصة الآتر الك، المصدر السابق.

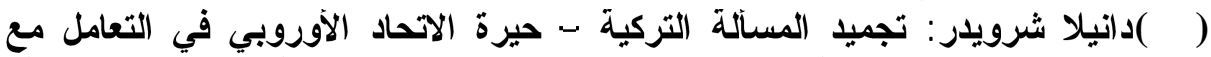

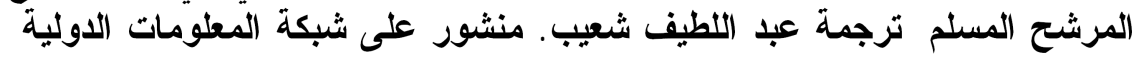
على الموقع الأكتروني:

http://www.qantara.da/webcom/show.artical.php

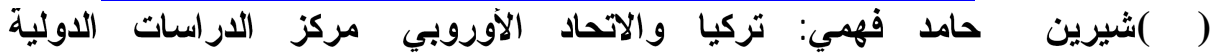

والاستر اتيجية، منشور على شبكة المعلومات الاولية، على الموقع الأكتروني: www./Islamonline.net/Arabic/shariha

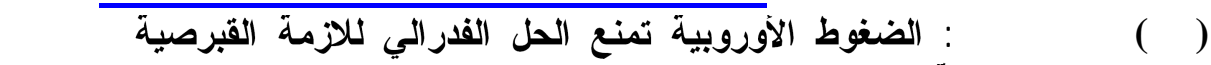

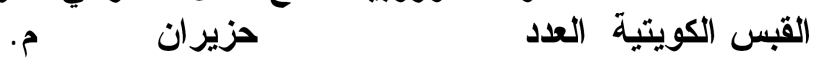
( ) عبد الجليل زيد المرهونه المصدر السابق.

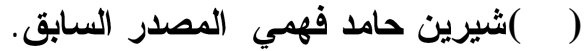

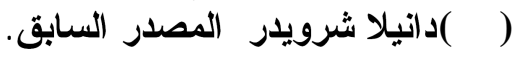

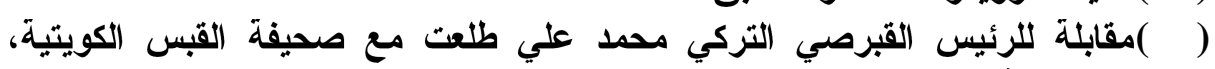

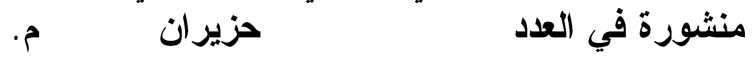
( ) علي العبد الله، المصدر السابق.

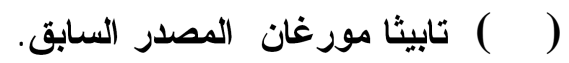


